\title{
Thermal conductivity of composites with nanoscale inclusions and size-dependent percolation
}

\author{
L H Liang ${ }^{1,2}$, Y G Wei ${ }^{1}$ and Baowen $\mathbf{L i}^{2,3}$ \\ ${ }^{1}$ LNM, Institute of Mechanics, Chinese Academy of Sciences, Beijing 100190, \\ People's Republic of China \\ ${ }^{2}$ Department of Physics and Centre for Computational Science and Engineering, \\ National University of Singapore, Singapore 117542, Singapore \\ ${ }^{3}$ NUS Graduate School for Integrative Sciences and Engineering, Singapore 117597, \\ Singapore \\ E-mail: lianglh@1nm.imech.ac.cn
}

Received 11 January 2008, in final form 14 May 2008

Published 12 August 2008

Online at stacks.iop.org/JPhysCM/20/365201

\begin{abstract}
An analytical model for thermal conductivity of composites with nanoparticles in a matrix is developed based on the effective medium theory by introducing the intrinsic size effect of thermal conductivity of nanoparticles and the interface thermal resistance effect between two phases. The model predicts the percolation of thermal conductivity with the volume fraction change of the second phase, and the percolation threshold depends on the size and the shape of the nanoparticles. The theoretical predictions are in agreement with the experimental results.
\end{abstract}

(Some figures in this article are in colour only in the electronic version)

Nanocomposites have a wide range of applications in the fields of optoelectronics, thermoelectrics, sensors, etc, due to their novel structure and properties [1-3], such as nonlinear optical properties of granular metal-dielectric composites [2] and the large enhancement of effective thermal conductivity of a fluid with the addition of a small amount of carbon nanotubes [3]. With continuous miniaturization of semiconductor and microelectronic devices, thermal transport and heat management problems have begun to attract a great deal of attention [4, 5]. Some devices such as computer processors and integrated circuits need high thermal conductivity, which is favorable for getting the heat away. On the other hand, for thermal barriers and thermoelectric devices, low thermal conductivity is desired. Studies have found that thermal conductivity of nanowires and thin films is size-dependent, it is lower than the corresponding bulk value [6]. However, there are not many theoretical studies on the thermal conductivity of nanocomposites, despite its importance in applications $[1,3]$.

The effective medium theory (EMT) is often used to study the physical properties of composites, such as dielectric constant [2], thermal conductivity and electrical conductivity [7]; the percolation of electrical conductivity is usually found, but there are few reports on the percolation of thermal conductivity. The role of interface thermal resistance (ITR) in the thermal conductivity of nanocomposites was emphasized in recent studies [8, 9], which improves the theoretical results based on the EMT, but the related work seems to be discussed only for the dilute limit [9]. Moreover, the intrinsic size effect of the thermal conductivity of nanoscale structures was not considered for composites based on the EMT. The study based on the Boltzmann transport equation considered both the interface and the size effects, but no percolation of thermal conductivity was found for nanocomposites [8]. In this paper, a general theoretical model about the effective thermal conductivity of nanocomposites in the whole range of volume fractions of the second phases is proposed based on the EMT by introducing both the intrinsic size effect and the ITR effect. The model predicts the percolation of the thermal conductivity for composites with nanoparticles or nanorods of smaller size.

Based on quantum scattering theory and the Green's function perturbation method, considering a composite medium whose thermal conductivity varies from point to point, the thermal conductivity $\kappa(r)$ corresponding to the point $r$ is expressed as a sum of a constant part of a homogeneous 
Table 1. The parameters in the model. In equation (4), $D_{0}=6 h$ and $4 h$ for nanoparticles and nanorods, respectively, $\alpha=2 S_{\mathrm{v}} /(3 R)+1$ and $S_{\mathrm{v}}=S_{\mathrm{m}}-R$ for semiconductors [15] with the melting entropy $S_{\mathrm{m}}=H_{\mathrm{m}} / T_{\mathrm{m}}, H_{\mathrm{m}}$ the melting enthalpy and $T_{\mathrm{m}}$ the melting temperature.

\begin{tabular}{llllllll}
\hline & $\kappa_{b}$ & \multicolumn{3}{c}{$H_{\mathrm{m}}$} \\
& $\left(\mathrm{W} \mathrm{m}^{-1} \mathrm{~K}^{-1}\right)$ & $h$ & $\left(\mathrm{KJ} \mathrm{mol}^{-1}\right)$ & $T_{\mathrm{m}}(\mathrm{K})$ & $v\left(\mathrm{~m} \mathrm{~s}^{-1}\right)$ & $C\left(10^{6} \mathrm{~J} \mathrm{~m}^{-3} \mathrm{~K}^{-1}\right)$ & $\rho\left(\mathrm{g} \mathrm{cm}^{-3}\right)$ \\
& {$[16]$} & $(\mathrm{nm})[17]$ & {$[16]$} & {$[16]$} & {$[18]$} & {$[16]$} & {$[16]$} \\
\hline $\mathrm{Si}$ & 148 & 0.3368 & 50.55 & 1685 & 2200 & 1.653 & 2.33 \\
$\mathrm{Ge}$ & 59.9 & 0.351 & 36.94 & 1210.4 & 5400 & 1.717 & 5.32 \\
\hline
\end{tabular}

medium $\kappa_{0}$ and an arbitrary fluctuating part $\kappa^{\prime}(r)$. By using the Green function $G$ for the homogeneous medium defined by $\kappa_{0}$ and the scattering matrix $T$ for the entire composite medium $\left(T=\kappa^{\prime}(r)+\kappa^{\prime}(r) G T=\kappa^{\prime}(r)(I+G T)\right.$ is related to the perturbation $\kappa^{\prime}(r)$ and $G, I$ is the unit tensor) [7], a rigorous solution for the temperature gradient distribution can be obtained. The resulting effective thermal conductivity $\kappa_{\mathrm{e}}$ of the composite is expressed as [7,9]

$$
\kappa_{\mathrm{e}}=\kappa_{0}+\frac{\langle T\rangle}{I+\langle G T\rangle},
$$

where \langle\rangle represents spatial averaging, and $\langle G\rangle$ and $\langle T\rangle$ are related to the thermal conductivity, the concentration and the geometry shape of each phase constituting the composite. According to the multiple-scattering approach, the matrix $T$ is given as [7, 9]

$$
\begin{aligned}
T= & \sum T_{n}+\sum_{m \neq n} T_{n} G T_{m}+\sum_{l \neq m \neq n} T_{n} G T_{m} G T_{l}+\cdots \\
& \cong \sum T_{n}=\sum \frac{\kappa_{n}^{\prime}}{I-G \kappa_{n}^{\prime}},
\end{aligned}
$$

where $T_{n}$ is the $T$ matrix of particle $n$, the first term on the right of the first part of the equation is the sum of the $T$ matrices of all particles and the successive terms represent the interaction among the particles (which is then neglected as a first-order approximation, considering the difficulty of obtaining exact solutions). $\kappa_{n}^{\prime}$ corresponds to $\kappa^{\prime}(r)$ of particle $n$. Substituting equation (2) into equation (1), and taking $\kappa_{0}$ to be the average value of thermal conductivity of each phase constituting the composite with the respective concentration weight, $\kappa_{\mathrm{e}}$ can be obtained, which is the average $t$-matrix approximation [7]. According to the coherent potential approximation based on the mean-field method, $\kappa_{0}$ can also be chosen so that the effect of the perturbation is as small as possible, $\kappa_{0}=\kappa_{\mathrm{e}}$ and $\langle T\rangle=0$ in the limit case, which is the self-consistent EMT [7]. Both approaches improve the results of the firstorder approximation.

According to the self-consistent EMT, the effective thermal conductivity $\kappa_{\mathrm{e}}$ of two-phase composites can be given by

$$
\left(1-V_{2}\right) \frac{\kappa_{1}-\kappa_{\mathrm{e}}}{L \kappa_{1}+(1-L) \kappa_{\mathrm{e}}}+V_{2} \frac{\kappa_{2}-\kappa_{\mathrm{e}}}{L \kappa_{2}+(1-L) \kappa_{\mathrm{e}}}=0,
$$

where $\kappa$ is the thermal conductivity, $V$ the volume fraction, the subscript 1 represents the matrix phase, 2 the inclusion particles, $L$ the depolarization factor related to the geometry shape with $T=\kappa^{\prime} /\left(1+L \kappa^{\prime} / \kappa_{0}\right)$ and $\left\langle\kappa^{\prime}\right\rangle=$ $\sum_{i=1,2} V_{i} \kappa_{i}^{\prime}[7,10]$. Note that the composites discussed here

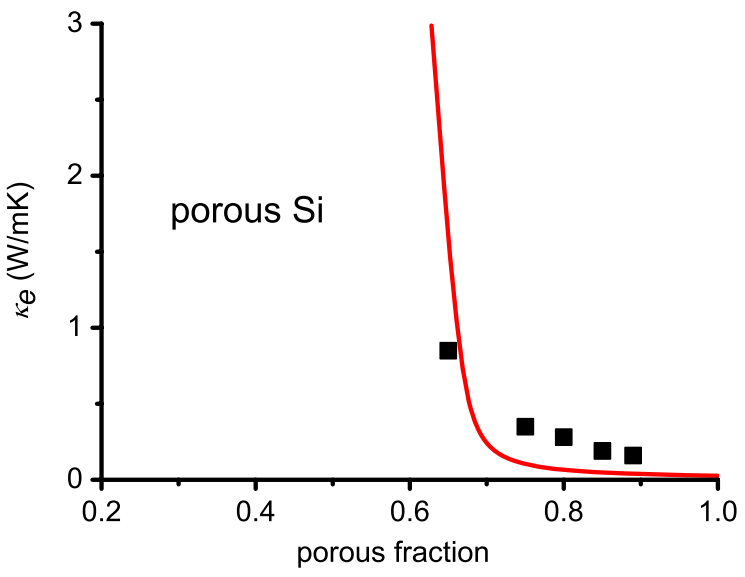

Figure 1. $\kappa_{\mathrm{e}}$ of porous Si. The symbols are experimental results [13], while the curve is a prediction based on the model. In equation (3), $\kappa_{1}=0.0262 \mathrm{~W} \mathrm{~m}^{-1} \mathrm{~K}^{-1}$ for air [14], $\kappa_{2}$ for $\mathrm{Si}$ and for the related parameters in equation (4) see table 1.

are isotropic and the particles are spherical, $L=1 / 3$ for both phases and each direction [7,9]. Generally, $L_{x}=L_{y}=$ $p^{2} /\left[2\left(p^{2}-1\right)\right]-p /\left[2\left(p^{2}-1\right)^{3 / 2}\right] \cosh ^{-1} p$ with the aspect ratio $p=a_{z} / a_{x}>1$ and the principal axis radii $a_{x}=a_{y}<a_{z}$, $L_{z}=1-2 L_{x}$, where the subscripts $x, y$ and $z$ denote the three principal axes [9]. For example, $L_{x}=0.5$ and $L_{z}=0$ for nanotubes with large $p$. Nan et al and Gao et al [11] have explained well the increased thermal conductivity of nanofluids with carbon nanotube filler using the EMT, although the filler fraction is very small and the conductivity difference between two phases is so large. For the inclusion particles in nanoscale, the size effect of the nanoparticles should contribute to the effective thermal conductivity of the composites. The intrinsically size-dependent thermal conductivity of nanoscale materials is given as

$$
\frac{\kappa_{2}}{\kappa_{2 \mathrm{~b}}}=\left[\exp \left(\frac{1-\alpha}{D / D_{0}-1}\right)\right]^{3 / 2}
$$

based on the kinetic formula of the bulk thermal conductivity by introducing the intrinsic size effects of phonon velocity and mean free path due to the phonon confinement [6], where $\kappa_{2 \mathrm{~b}}$ represents the bulk thermal conductivity of the second phase inclusions, $D$ the diameter of the nanoparticles, $\alpha=$ $2 S_{\mathrm{v}} /(3 R)+1$ is a material constant related to the lattice vibration with the vibrational entropy of melting $S_{\mathrm{v}}$ and the ideal gas constant $R[6,12], D_{0}=2(3-f) h$ is a critical size with atomic or molecular diameter $h$ and the dimension $f=0,1$ and 2 for nanoparticles, nanowires and thin films, respectively $[6,12]$. 

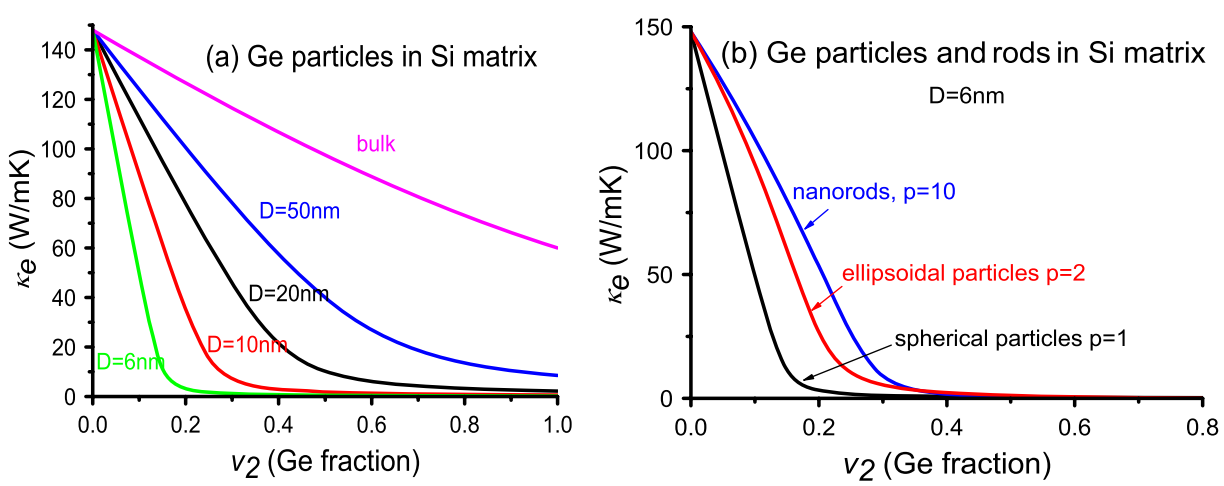

Figure 2. $\kappa_{\mathrm{e}}$ of SiGe composites based on equations (3)-(6). (a) Ge particles with diameter $D=6,10,20$ and $50 \mathrm{~nm}$ and the bulk, respectively. In equation (3), $\kappa_{1}$ for bulk $\mathrm{Si}, \kappa_{2}$ for Ge; in equation (6), $d=2 \mathrm{~nm}$ is assumed; the other related parameters are in table 1. (b) Ge particles and rods with diameters $D=6 \mathrm{~nm}$ and different aspect ratios $p=1,2$ and 10 . For rods, the length is $60 \mathrm{~nm}, p=10$, $L_{2 x}=L_{2 y}=0.5$ and $L_{2 z}=0, X=\frac{1}{(1+2 d / D)^{2}}$ in equation (5), and the sum of $x$ and $y$ directions is considered in equation (3), $\sum_{j=x, y, z}\left(1-V_{2} / X\right) \frac{\kappa_{1}-\kappa_{\mathrm{e}}}{L_{1 j} \kappa_{1}+\left(1-L_{1 j}\right) \kappa_{\mathrm{e}}}+\sum_{j=x, y, z}\left(V_{2} / X\right) \frac{\kappa_{\mathrm{c}}-\kappa_{\mathrm{e}}}{L_{2 j} \kappa_{\mathrm{c}}+\left(1-L_{2 j}\right) \kappa_{\mathrm{e}}}=0, L_{1 x, 1 y, 1 z}=1 / 3$. The other calculation is the same as (a).

Figure 1 shows $\kappa_{\mathrm{e}}$ of porous Si by considering the intrinsic size effect of Si nanoparticles and substituting equation (4) into equation (3). The porous $\mathrm{Si}$ is considered as a composite of the pores (air) and the Si particles. $D=8.5 \mathrm{~nm}$ is taken in equation (4) since the size range of Si particles is $8.5-13 \mathrm{~nm}$ in the experiment [13], and there is no obvious difference among the calculated results in this size range. It can be seen from the figure that the prediction agrees with the experimental result in the tendency: $\kappa_{\mathrm{e}}$ decreases nonlinearly with increasing porosity, when the porous fraction increases to about $0.7, \kappa_{\mathrm{e}}$ is notably smaller and the rate of decrease becomes slower, the porous $\mathrm{Si}$ is almost a thermal insulator, and $\kappa_{\mathrm{e}}$ shows the percolation, i.e. the abrupt transition of the system's property from the conduction to the insulation state. Note that, when the porosity is larger than 0.7 , the experimental data are larger than the theoretical predictions. The reason may be that the fabrication of porous Si with the higher porosity $(>0.75)$ was related to the oxidation of the porous $\mathrm{Si}$ with the lower porosity and the subsequent removing of the oxide. In the process the plastic deformation of the Si particles occurred, which induced the increased conduction percolation strength [13].

On the other hand, the interface thermal resistance between two phases, especially two solid phases, should contribute to the effective thermal conductivity of the composites, but this interface effect is not included in equation (3). Therefore, we further assume that there is a interface layer between two phases, and take the inclusion particle with the interface layer as a composite unit. Then we replace $\kappa_{2}$ by the thermal conductivity $\kappa_{\mathrm{c}}$ of the composite unit in equation (3) and replace $V_{2}$ by the effective volume fraction $V_{2} / X$ of the composite unit in the equation, so the interface effect can be included by introducing the interface layer, where $X=\frac{1}{(1+2 d / D)^{3}}$ is the interface ratio factor and $d$ is the thickness of the interface layer [7]. The thermal conductivity $\kappa_{\mathrm{c}}$ of the composite unit is expressed as

$$
\kappa_{\mathrm{c}}=\kappa_{l} \frac{2 \kappa_{l}+\kappa_{2}+2 X\left(\kappa_{2}-\kappa_{l}\right)}{2 \kappa_{l}+\kappa_{2}-X\left(\kappa_{2}-\kappa_{l}\right)}
$$

based on the average $t$-matrix approximation by taking the interface layer as phase 1 and the particle as phase 2, where $\kappa_{l}$ is the thermal conductivity of the interface layer and $X$ reflects the volume fraction of the particle in the composite unit. $\kappa_{l}$ can be calculated by multiplying the interface thermal conductance $G$ by $d, G=\frac{t v C}{4}, t$ is the interfacial phonon transmission coefficient, and $v$ and $C$ the average phonon velocity and the specific heat of two phases, respectively [19]. Therefore,

$$
\kappa_{l}=G d=\frac{t v C d}{4} .
$$

The interfacial phonon transmission can be calculated based on the acoustic mismatch model, $t=\frac{4 Z_{1} Z_{2}}{\left(Z_{1}+Z_{2}\right)^{2}}$, where $Z=\rho v$ is the acoustic impedance and $\rho$ is the mass density [4, 20]. Note that, although the interface scattering term is not included in equation (4), the interface thermal resistance effect is included in equations (6) and (5), and there is no obvious difference in the obtained interface resistance values between the diffusive mismatch model (scattering limit) and the acoustic mismatch model for solidsolid interfaces [20].

Substituting equations (4) and (6) into equation (5), respectively, then equation (5) into equation (3), the thermal conductivity of composites can be calculated by considering both the size and the interface effects. Figure 2(a) shows $\kappa_{\mathrm{e}}$ of the composites with Ge nanoparticles dispersed in an Si matrix. It can be found that $\kappa_{\mathrm{e}}$ decreases with increasing fraction $V_{2}$ and the reducing size $D$ of Ge particles, which is reasonable since the thermal conductivity of Ge is lower than that of $\mathrm{Si}$ and $\kappa_{2}$ decreases with the reducing $D$ in terms of equation (4). Moreover, the nonlinear behavior of $\kappa_{\mathrm{e}}$ becomes obvious with the reducing $D$ of $\mathrm{Ge}$; when $D<10 \mathrm{~nm}$, the percolation behavior appears, i.e. when $V_{2}$ increases to a certain fraction corresponding to the percolation threshold (the volume fraction at which the abrupt transition of the system's property occurs), $\kappa_{\mathrm{e}}$ becomes very low and the composites become almost thermal insulators due to the large contribution of the smaller-sized Ge with low thermal conductivity. Also the percolation threshold decreases with the reducing size of Ge nanoparticles. Figure 2(b) shows the effect of the aspect ratio of Ge nanofillers. It can be seen that $\kappa_{\mathrm{e}}$ increases with the increasing aspect ratio of $\mathrm{Ge}$ at the same volume fraction 


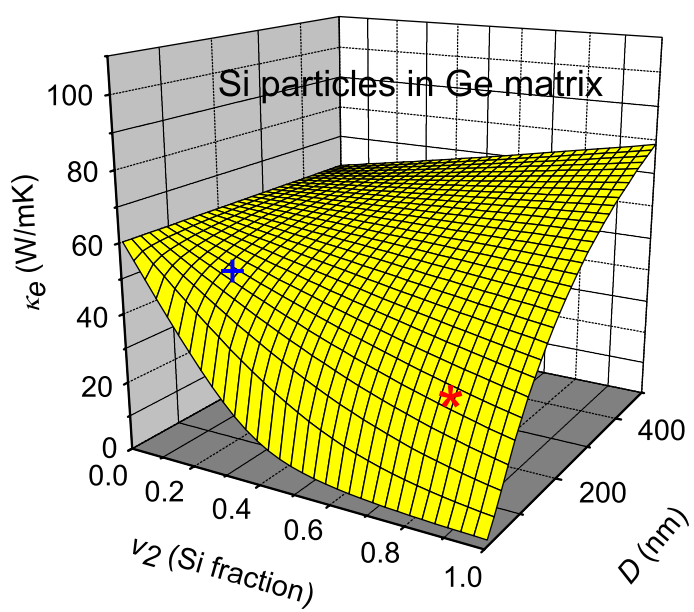

Figure 3. The same calculation as in figure 2(a) except for the exchange of roles of $\mathrm{Si}$ and $\mathrm{Ge}$ in the equations. The star denoting $\kappa_{\mathrm{e}}$ for $\mathrm{Si}_{80} \mathrm{Ge}_{20}$ and the cross for $\mathrm{Si}_{20} \mathrm{Ge}_{80}$ with $\mathrm{Si}$ particles of $100 \mathrm{~nm}$ are marked to compare with the experimental results [21].

and the same diameter, which is reasonable since the volume of a nanorod with the larger aspect ratio is larger than that of a nanoparticle with the same diameter, the number of nanorods is smaller than that of nanoparticles with the same volume fraction and the contribution to the decrease of thermal conductivity of the bulk Si matrix from Ge nanorod fillers is smaller than that from the nanoparticle fillers. Also the percolation threshold increases with the increasing aspect ratio of nanofillers. Note that $\kappa_{\mathrm{e}}$ of composites with nanofillers of the aspect ratio larger than one may be anisotropic depending on the orientation distribution of the nanofillers in the matrix. The randomly oriented nanorod filler is discussed here simply.

Figure 3 shows how $\kappa_{\mathrm{e}}$ of the composites with $\mathrm{Si}$ nanoparticles in a Ge matrix changes with the fraction $V_{2}$ and the size $D$ of $\mathrm{Si}$, which is different from that for $\mathrm{Ge}$ nanoparticles in Si. Although the thermal conductivity still decreases with the reducing $D$ of $\mathrm{Si}, \kappa_{\mathrm{e}}$ does not always increase with the increasing fraction of $\mathrm{Si}$, which depends on the size of Si. Only for the composites with Si particles larger than about $280 \mathrm{~nm}$ does $\kappa_{\mathrm{e}}$ increase with increasing $V_{2}$ due to the higher thermal conductivity of the large-sized Si compared to Ge. For Si particles with $D<280 \mathrm{~nm}, \kappa_{\mathrm{e}}$ decreases with the increasing fraction of $\mathrm{Si}$, which is in agreement with the experimental result: the thermal conductivity of $\mathrm{Si}_{80} \mathrm{Ge}_{20}$ nanocomposites with $\mathrm{Si}$ particles of $100 \mathrm{~nm}$ is lower than that of $\mathrm{Si}_{20} \mathrm{Ge}_{80}$ with the same-sized Si nanoparticles [21].

In conclusion, a simple model of thermal conductivity of nanocomposites is established. The model predicts the percolation of thermal conductivity for the composites with the smaller-sized nanoparticles or nanorods in a bulk matrix and for the nanoporous medium, which is understandable since the thermal conductivity of the smaller-sized nanofillers or air is very low and the difference between two phases constituting the composites is large. It can be deduced that if two phases of composites are both in nanoscale or the difference between two phases is small, the percolation behavior will weaken. Nevertheless, the percolation behavior can appear not only in electrical transport but also in heat transport, depending on the corresponding conduction network formation determined by the structure of the composites. The model gives a general guide for the fabrication and the application related to heat problems by designing the constituent, the size, and the structure of nanocomposites.

\section{Acknowledgments}

The work is supported by grants partly from the Faculty Research Grant of NUS, partly from the Chinese Academy of Sciences through grant KJCX2-YW-M04 and from the NSFC through grant nos. 10721202 and 10432050.

\section{References}

[1] Venkatasubramanian R, Siivola E, Colpitts T and O’Quinn B 2001 Nature 413597

Costescu R M, Chill D G, Fabreguette F H, Sechrist Z A and George S M 2004 Science $\mathbf{3 0 3} 989$

[2] Gao L, Jones T K W, Yu K W and Li Z Y 2000 J. Phys.: Condens. Matter 126825

Gao L, Yu K W, Li Z Y and Hu B 2001 Phys. Rev. E 64036615

[3] Choi S U S, Zhang Z G, Yu W, Lockwood F E and Grulke E A 2001 Appl. Phys. Lett. 792252

Biercuk M J, Llaguno M C, Radosavljevic M, Hyun J K, Johnson A T and Fisher J E 2002 Appl. Phys. Lett. 802767

[4] Cahill D G, Ford W K, Goodson K E, Mahan G D, Majumdar A, Maris H J, Merlin R and Phillpot S R 2003 J. Appl. Phys. 93793

[5] Au Yeung T C, Chiam T C, Sun C Q, Gu M, Shangguan W Z and Kam C H 2005 J. Appl. Phys. 98113707

Au Yeung T C, Gu M X, Sun C Q, Chen G C K, Wong D W K and Nosik V 2006 Phys. Rev. B 74155317 Yu B and Li B 2006 Phys. Rev. E 73066302

[6] Liang L H and Li B 2006 Phys. Rev. B 73153303 and references therein

[7] Nan C W 1993 Prog. Mater. Sci. 371

[8] Yang R-G, Chen G and Dresselhaus M S 2005 Phys. Rev. B 72125418

Tian W-X and Yang R-G 2007 Appl. Phys. Lett. 9263105

[9] Nan C W, Birringer R, Clarke D R and Gleiter H 1997 J. Appl. Phys. 816692

[10] Hori M 1977 J. Math. Phys. 18487

[11] Nan C W, Shi Z and Lin Y 2003 Chem. Phys. Lett. 375666

Gao L, Zhou X F and Ding Y L 2007 Chem. Phys. Lett. 434297

[12] Shi F G 1994 J. Mater. Res. 51307

Jiang Q, Shi H X and Zhao M 1999 J. Chem. Phys. 1112176

[13] Chantrenne P and Lysenko V 2005 Phys. Rev. B 72035318

[14] Lide D R 2004-2005 Handbook of Chemistry and Physics 85th edn (Boca Raton, FL: Chemical Rubber Company) pp 6-212

[15] Zhang Z, Zhang M and Jiang Q 2001 Semicond. Sci. Technol. 16 L33

[16] Periodic Table of the Elements 1980 (Skokie, IL: Sargent-Welch Scientific Company) p 1

[17] King H W 1983 Physical Metallurgy 3rd edn, ed R W Cahn and P Haasen (Amsterdam: North-Holland) pp 63-5

[18] Winter M 1993-2006 WebElements, the Periodic Table on the $W W W$ (Sheffield: The University of Sheffield and WebElements Ltd)

[19] Chen Y-F, Li D-Y, Yang J-K, Wu Y-H, Lukes J R and Majumdar A 2004 Physica B 349270

[20] Khalatnikov I M 1952 Zh. Eksp. Teor. Fiz. 22687 Swartz E T and Pohl R O 1989 Rev. Mod. Phys. 61605

[21] Lee H 2005 Experimental study of thermal conductivity reduction of silicon-germanium nanocomposites for thermoelectric application Master Thesis Massachusetts Institute of Technology p 57 